\title{
Physiologic Predictors of Lumbar Spine Bone Mass in Neonates
}

\author{
WINSTON W. K. KOO AND ELAINE M. HOCKMAN \\ Departments of Pediatrics, Obstetrics and Gynecology, University of Tennessee, Memphis, TN, U.S.A. \\ [W.W.K.K], and Computing and Information Technology, Wayne State University, \\ Detroit, MI, U.S.A. [E.M.H.]
}

\begin{abstract}
Dual energy x-ray absorptiometry (DXA) of the lumbar spine (LS) was measured in 201 singleton infants with birth weights from 1152 to $3970 \mathrm{~g}$ and gestational ages from 27 to $42 \mathrm{wk}$. All infants were well and studied at a mean $( \pm \mathrm{SD})$ of $2.1( \pm 1.6)$ days after birth. There were 75 Caucasian (46 males, 29 females) and 126 African American infants (58 males, 68 females). Scan acquisition of the first to fourth lumbar vertebrae was performed with a single beam whole body scanner (Hologic QDR 1000/W densitometer, Hologic Inc, Waltham, MA, U.S.A.) using the infant spine mode. Scan analysis was performed with software version 4.57Q and consistent region of interest. The SD of difference for duplicate LS scans is $<1.4 \%$ at a mean bone mineral content (BMC) of $2.14 \mathrm{~g}$. Results show that LS BMC, area, bone mineral density (BMD) increased by approximately $550 \%, 280 \%$ and $180 \%$, respectively, between 27 and $42 \mathrm{wk}$ gestational age. Body mass accounted for about $70 \%$ and $55 \%$ of the variance in BMC and BMD respectively. In contrast, the infant's length appears to be the best determinant of LS area and
\end{abstract}

\section{ABSTRACT}

accounts for about $75 \%$ of the variance in LS area. Race, gender or season has little or no effect on LS bone mass. There was progressive increase in BMC and area from first to fourth lumbar vertebra but BMD was significantly higher only at the fourth lumbar vertebra. We conclude that DXA LS can be performed even in small preterm infants. Its excellent precision, low radiation exposure and rapid scan acquisition offers promise as a useful tool for widespread use in pediatrics. Our data may be used as a basis for further studies in physiologic and pathologic situations that may affect bone mineralization in infants. (Pediatr Res 48: 485-489, 2000)
Abbreviations
DXA, dual energy x-ray absorptiometry
LS, lumbar spine
BMC, bone mineral content
BMD, bone mineral density

Understanding of the physiologic development of skeletal mineralization in infants is now possible since the validation of dual energy x-ray absorptiometry (DXA) for the measurement of bone mineral status of the whole body $(1,2)$ and lumbar spine (3). We reported that body weight is the major determinant of total body bone mass at birth (4) and postnatally (5) but there is no data to document the extent to which these and other physiologic variables determine the bone mineral status at the vertebrae. The aims of this study were to document differences in bone mass at the lumbar spine at different birth weights and gestational ages using DXA measurement at the lumbar spine (LS). In addition, we aim to determine the predictive value of anthropometric measurements, gestational age, race, gender, and season on DXA LS.

Received November 12, 1999; accepted June 13, 2000.

Correspondence: Winston Koo, Department of Pediatrics, Hutzel Hospital, 4707 St Antoine Boulevard, Detroit, MI 48201, U.S.A.

Supported by a University of Tennessee Medical Research Grant and by The University of Tennessee - Memphis Clinical Research Center, USPHS grant RR 00211-29.

No reprints available.

\section{MATERIALS AND METHODS}

Subjects. The total study population included 201 singleton infants with birth weights from 1152 to $3970 \mathrm{~g}$. The subjects' birth weights were between 10th and 90th percentiles for gestational ages (6). Gestational ages of the subjects as determined by maternal menstrual dating and/or ultrasound were from 27 to $42 \mathrm{wk}$ and within $2 \mathrm{wk}$ of gestational age assessment by standard examination (7). 76 subjects were preterm with gestational age $<38 \mathrm{wk}$, of whom 53 subjects had low birth weight ( $\leq 2500 \mathrm{~g}$ ). There were 75 Caucasian (42 term, 46 males, 29 females) and 126 African American infants (83 term, 58 males, 68 females). All infants were clinically well at the time of study with resolution of any acute illnesses including the need for mechanical ventilatory support or acute medical therapy. None of the subjects had a family history of disorders that may affect skeletal development or preexisting chronic maternal illness. This study was approved by the Institutional Review Board for human subjects at the University of Tennessee-Memphis, and written informed consent was obtained from each subject's parent. 
Anthropometric measurements. The nude weight of the infant and the weight of cotton blanket that swaddled the infant, and length of the infant were measured for each study. The study weight is the sum of the nude weight and the blanket weight. The weight in grams was determined with a digital electronic scale (Air Shields, Vickers, OH, U.S.A.). The scales were regularly maintained by the hospital Biomedical Instrumentation personnel and calibrated with known standard weights. Recumbent length was the average of two consecutive measurements within $0.4 \mathrm{~cm}$ and was determined using a standard length board (Ellard Instrumentation Ltd, Seattle, WA, U.S.A.).

DXA measurements. DXA scans were performed at a mean $( \pm \mathrm{SD})$ of $2.1( \pm 1.6)$ days after birth. Ten subjects were studied between 6 and $7.7 \mathrm{~d}$ after they had recovered from transient respiratory illnesses. Scan acquisition of the first to fourth lumbar vertebrae (LS) was performed with a single beam whole body scanner (Hologic QDR 1000/W densitometer, Hologic Inc, Waltham, MA, U.S.A.) using the commercial infant spine mode. Specifically, scan acquisition used the fast spine mode and fixed scan dimension of $10-\mathrm{cm}$ length and 8 -cm width. Each scan was completed in one to two minutes depending on size of the infant and may be less than one minute in very low birth weight infants. The x-ray beam collimation was $3.2 \mathrm{~mm}$ and the measured entry radiation exposure was $10 \mathrm{uSv}$ (1 mrem) using a Radcal Corporation model 1515 radiation monitor (MDH Company, Monrovia, CA, U.S.A.). All scans for this study were performed with the subject placed on top of the infant platform with a cotton blanket interposed between the subject and the platform (1). Each subject was swaddled in a cotton blanket during the scanning. A heat lamp was used as needed to maintain a satisfactory environmental temperature. Occasionally the scanning procedure was interrupted if movement artifact was noted, and a repeat scan was performed when the infant had been pacified.

Scan analyses were performed using the software (Version 4.57Q) developed in conjunction with the manufacturer. The region of interest includes an area equivalent to one vertebral width on either side of the first to fourth lumbar vertebrae en-bloc. The software allowed for analysis of first to fourth lumbar vertebrae en bloc and also for individual lumbar vertebra. Delineation of individual vertebra was performed by the operator according to manufacturer's recommendation using the same technique as that for adult LS scans, although quantification of bone mass was based on an ultra high-resolution analysis that has a lower bone detection threshold suitable for use in infants.

Long-term $(>3 \mathrm{y})$ coefficients of variation $(\mathrm{CV})$ for the determination of bone mineral content (BMC), bone area (area), and bone mineral density (BMD), using an anthropomorphic spine phantom is $<0.31 \%$ for all parameters. The average annual rate of change for each of these measurements was not significantly different from zero. The in vivo replication of DXA measurements of lumbar spines of 49 infants was highly significantly correlated $(\mathrm{r}=0.99$ and $p<0.001$ for all parameters, i.e. $\mathrm{BMC}$, area, and $\mathrm{BMD}$, respectively). The $\mathrm{SD}$ (SD) of difference (8) between paired DXA measurements in these infants for LS BMC was $<1.4 \%$ at a mean of $2.14 \mathrm{~g}$, LS area was $<2.8 \%$ at a mean of $8.8 \mathrm{~cm}^{2}$, and LS BMD was $<0.4 \%$ at a mean of $0.227 \mathrm{~g} / \mathrm{cm}^{2}$, respectively. Whereas, the SD of difference between duplicated pairs of LS scans for BMC, area and BMD increased to $8.5 \%, 13.9 \%$, and $9.6 \%$, respectively, if one of the scans had movement artifacts. Normative data were reported only for scans without movement artifact.

Statistical analyses. A principal component factor analysis showed that the three measures of weight (birth weight, study weight, study nude weight) were highly interrelated with loadings of 0.994 to 0.998 . A composite weight score was computed that accounts for $99.7 \%$ of the variance. Thus any of the weight variables can be used in the regression analysis with equal validity. Nude weight was used in all analyses to minimize the entry of multiple collinear independent weight variables in the analyses. Its use has other advantages since it is the most consistent weight measurement without concern for the varied amount of clothing and covering, and it also provides consistency with our previous publications $(4,5)$.

Stepwise multiple linear regression analyses were performed to determine the explanatory ability of each independent variable known to have the potential to affect growth and bone mineralization: gestational age, postnatal age at study, nude weight, length, race, gender, and season of birth, on each of the three dependent variables (BMC, area, and BMD) separately. The seasonal variable was determined by coding the month of birth at 3 monthly intervals beginning at March as spring, and was transformed into dummy variables using spring as the reference season.

For each of the dependent variables, a final model predictive equation was generated, containing only statistically significant independent variables. This represents a hierarchical modeling process that first determines the most powerful individual predictor of bone mineral status and then determines if any other set(s) of independent variable(s) either augmented our initial model's explanatory ability or diminished the explanatory capability of the single best predictor.

Repeated measures analysis of variance (ANOVA) with planned orthogonal contrasts was performed to ascertain differences in the three DXA dependent variables (BMC, area, BMD) at each of the four lumbar vertebrae.

All statistical tests were performed with SPSS 10.0 (SPSS Inc, Chicago, IL, U.S.A.) for windows at an adopted significance level of 0.05 .

\section{RESULTS}

LS bone mineral content (BMC), area, and bone mineral density (BMD) increased by approximately $550 \%, 280 \%$, and $180 \%$, respectively, between 27 and 42 wk gestational age. The mean regression line and $95 \%$ confidence interval for individual BMC, BMD and area values as a function of birth weight or length are shown are shown in Figs. 1a, 1b, and 2.

Our data show that body weight systematically heavily dominated the model's explanatory power for LS BMC and $\mathrm{BMD}$, whereas, length is the dominant predictor of LS area. However, body weight becomes the dominant predictor for LS 


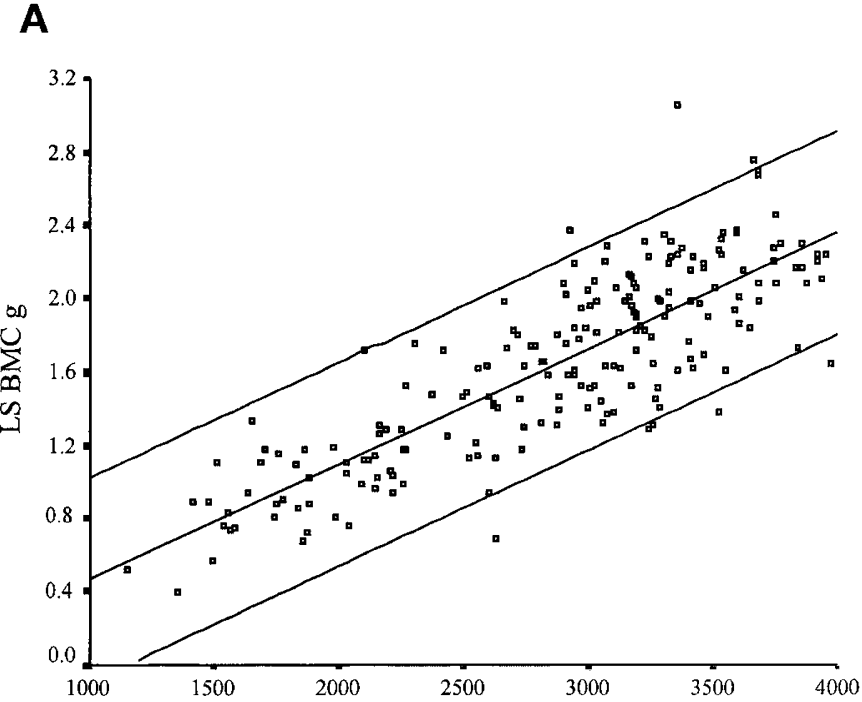

Birth weight $g$

B

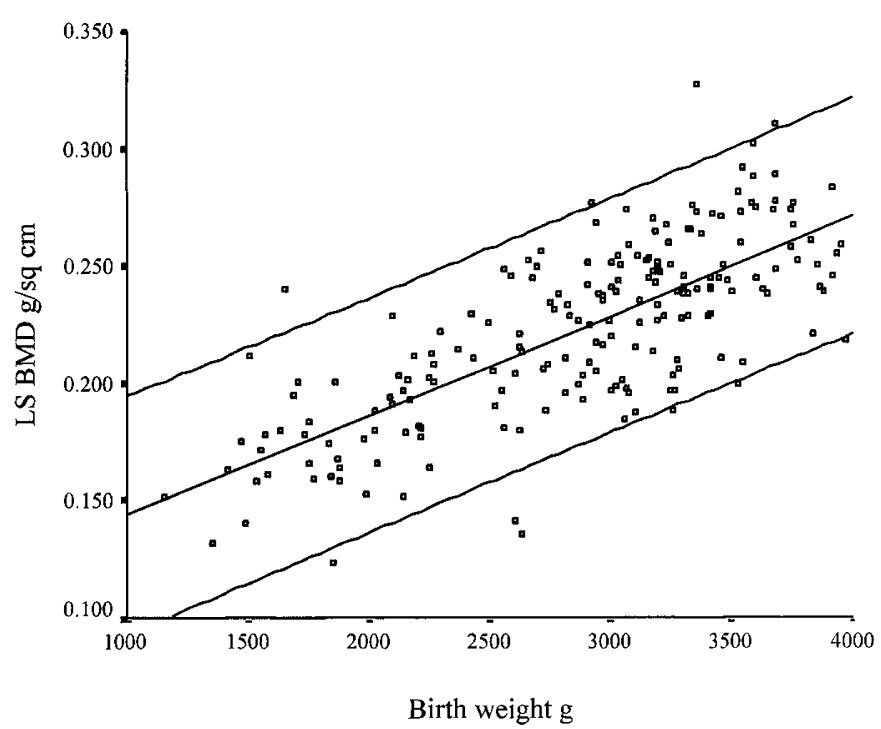

Figure 1. Scatter plot including mean regression line and $95 \%$ confidence interval for $(A)$ bone mineral content (LS BMC) and $(B)$ bone mineral density (LS BMD) at first to fourth lumbar vertebrae as a function of birth weight

area with an adjusted $\mathrm{R}^{2}$ of 0.72 if length was excluded from the regression. Other independent variables entered into the model have minimal or no predictive value on LS bone mineral status (Table 1).

The final regression equations for the prediction of LS DXA measurements including all statistically significant predictors are:

$D X A_{\text {LSBMC }}=-2.049+3.775 \mathrm{E}-04$ Nude weight $\mathrm{g}+$

0.05971 Length $\mathrm{cm}-0.1$ Race

$D X A_{\text {LSarea }}=-9.466+0.278$ Length $\mathrm{cm}$

+0.01376 GA days

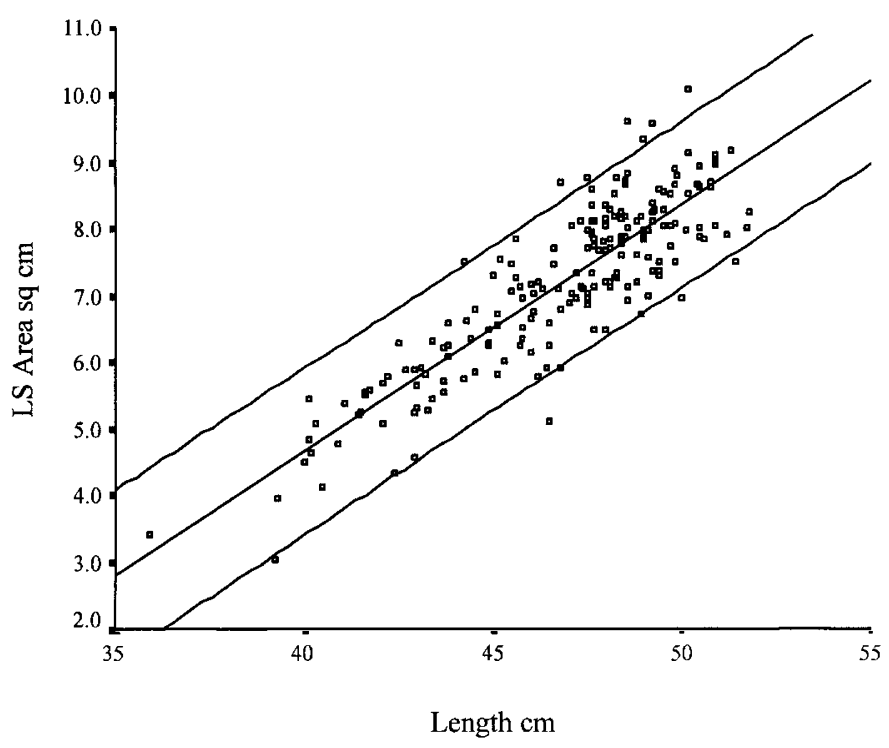

Figure 2. Scatter plot including mean regression line and 95\% confidence interval for first to fourth lumbar vertebrae area (LS area) as a function of length

Table 1. Regression Model Containing the Statistically Significant Independent Variables in the Prediction of Dual Energy X-Ray Absorptiometry Derived Lumbar Spine Bone Mineralization in Neonates

\begin{tabular}{llccc}
\hline \multirow{2}{*}{ Dependent Variable } & \multicolumn{1}{c}{$\begin{array}{c}\text { Independent } \\
\text { Variable }\end{array}$} & $\begin{array}{c}\text { Standardized } \\
\text { Coefficient-beta }\end{array}$ & $p$ & $\begin{array}{c}\text { Adjusted } \\
\mathrm{R}^{2}\end{array}$ \\
\hline Bone mineral content & Nude weight & 0.509 & $<0.001$ & 0.688 \\
& Length & 0.352 & 0.001 & 0.704 \\
Area & Race* & -0.097 & $<0.05$ & 0.712 \\
\multirow{2}{*}{ Bone mineral density } & Length & 0.653 & $<0.001$ & 0.754 \\
& Gestational age & 0.245 & 0.001 & 0.766 \\
& Nude weight & 0.750 & $<0.001$ & 0.549 \\
& Race* & -0.147 & $<0.01$ & 0.569 \\
\hline
\end{tabular}

* Caucasian neonates have higher BMC and BMD.

$D X A_{\text {LSBMD }}=0.125+4.173$ E-05 Nude weight $\mathrm{g}$

-0.0112 Race

where Race $=1$ for Caucasian and 2 for African American.

The proportional distribution of area, BMC, and BMD at each vertebra is shown in Table 2A. Planned contrasts from ANOVA show a progressively higher mean BMC and area from first to the fourth vertebra. However, mean BMD was significantly higher only at the fourth vertebra as shown in Table 2B. A sample size as small as 89 subjects would have been sufficient to detect a small effect size at a significance of 0.01 and a power of 0.80 .

\section{DISCUSSION}

DXA measurement of LS has a number of advantages over the current DXA measurement of the whole body particularly in sick infants. Its ability to accurately measure small amounts of hydroxyapatite comparable to the low bone mass in neonatal spine using the same DXA technique as that used in this study has been reported (3). Rapid scan acquisition at LS with a 3- to 5 -fold shorter scanning time compare with whole body scan significantly lowers the risk of artifacts from movement (9). 
Table 2. Bone Mineral Content (BMC), Area, and Bone Mineral Density (BMD) of Each Lumbar Vertebra as a Percentage of Dual Energy X-Ray Absorptiometry Measurements at First to Fourth Lumbar Vertebrae in 201 Neonates

\begin{tabular}{|c|c|c|c|}
\hline \multicolumn{4}{|c|}{ A) Percentage of Combined First to Fourth Lumbar Vertebrae Values, Mean \pm SD } \\
\hline Lumbar Vertebra & $\mathrm{BMC}$ & Area & BMD \\
\hline First & $21.9 \pm 3.4$ & $22.2 \pm 3.2$ & $99.8 \pm 11.0$ \\
\hline Second & $23.9 \pm 2.9$ & $24.4 \pm 2.4$ & $98.0 \pm 7.9$ \\
\hline Third & $25.5 \pm 2.7$ & $25.8 \pm 2.4$ & $98.8 \pm 7.0$ \\
\hline Fourth & $28.6 \pm 4.0$ & $27.7 \pm 3.3$ & $103.4 \pm 7.7$ \\
\hline
\end{tabular}

B) Repeated Measures Contrasts

\begin{tabular}{|c|c|c|c|c|c|c|}
\hline \multirow[b]{2}{*}{ Lumbar Vertebra } & \multicolumn{2}{|c|}{ BMC g } & \multicolumn{2}{|c|}{ Area $\mathrm{cm}^{2}$} & \multicolumn{2}{|c|}{$\mathrm{BMD} \mathrm{g} / \mathrm{cm}^{2}$} \\
\hline & $\mathrm{F}$ & $p$ & $\mathrm{~F}$ & $p$ & $\mathrm{~F}$ & $p$ \\
\hline Second vs First & 39.7 & $<0.001$ & 53.1 & $<0.001$ & 1.2 & NS \\
\hline Third vs Previous & 88.1 & $<0.001$ & 117.8 & $<0.001$ & 0.0 & NS \\
\hline Fourth vs Previous & 162.312 & $<0.001$ & 126.6 & $<0.001$ & 38.1 & $<0.001$ \\
\hline
\end{tabular}

Clinical usefulness of DXA LS measurement is further supported by the excellent repeatability of duplicate scans in large numbers of infants. Low radiation exposure from DXA LS is comparable to DXA whole body measurements $(4,5)$. Thus DXA LS has the potential for widespread application to further understand the physiologic and pathologic determinant of bone mineralization and may be a useful tool for longitudinal studies in pediatrics.

Our subjects were recruited from an urban tertiary health care facility typical of most cities in United States with a large population of African American. All infants were clinically well with no significant maternal or family history of bone disorder, they included subjects of both gender and different racial origin and from a wide range of gestation. Thus, our data may be considered as clinically representative of LS bone mass measurements in normal neonates, and the sample size for this study was more than adequate for detection of significant relationships with adequate statistical power. These in vivo measurements of LS bone mass are important to the understanding of physiologic variables that might affect bone mineralization in infants, and may allow the development of therapeutic goals in pathologic conditions with low bone mass, for example, in infants with congenital defects such as osteogenesis imperfecta (10) or acquired defects such as osteopenia of prematurity (11).

In this study, we demonstrated that body mass is the major determinant of LS bone mineral status. This is consistent with our previous reports that body mass is the major determinant of whole body bone mineral status $(4,5)$. However, body mass accounts for only about $70 \%$ and $55 \%$ of the variance in LS $\mathrm{BMC}$ and LS BMD respectively and is lower than that reported with whole body DXA measurements. Addition of the infant's length to the model equation improved the predictive effect by $\sim 2 \%$ presumably because length and weight tend to be collinear at birth. The relatively low BMC at the lumbar vertebrae (average $\sim 2.9 \%$ of the whole body) and other as yet undefined factors may account for the lower predictive value of body mass for LS BMC.

It is not surprising that body mass alone has a lower predictive value for LS BMD than for LS BMC since it is an areal density calculated by LS BMC/LS area and changes in LS $\mathrm{BMC}$ and LS area for infants at various birth weights and lengths are not completely proportional. Our data supports the previous reports from us $(4,5,12)$ and others $(13)$ that caution is needed in the interpretation of areal BMD in the growing skeleton. Whether body mass may have better predictive value for true volumetric density measurement of the LS is not known.

In contrast, the infant's length appears to be the best determinant of LS area and accounts for as much as $75 \%$ of the variance in LS area. However, body weight becomes the dominant predictor of LS area if length was excluded from the regression model. This is presumably due to their colinearity in the newborn infant. Our data are consistent with the twodimensional nature of DXA area measurement, where vertebral height is primarily responsible for trunk length. The relatively small area of lumbar vertebrae (average $\sim 2.6 \%$ of whole body skeletal area) may explain the inability for length to account for total variance of LS area measurements.

This study show that the extent of racial effect on LS bone mass in infants is probably quite modest once body mass and length are taken into account. Our data are consistent with previous reports on the absence of racial and gender effect on skeletal weight, density and percent ash (14), total body DXA measurements $(4,5)$ and distal radial BMC $(15)$ of neonates. However, racial and gender effect on bone mineral status is well described for older age groups. For example, blacks have greater BMD than whites $(14,16,17)$ and males have greater BMD than females $(17,18)$ beginning at pubescence. Thus, the racial and gender effect on bone mineral status appears to be age dependent. The lack of seasonal effect on LS bone mineral status is consistent with our earlier report on DXA whole body measurements in infants $(4,5)$. The performance of DXA studies soon after birth is likely to have accounted for the lack of influence on LS bone mineral status from postnatal age.

We believe this is the first description of in vivo quantitative differences among various lumbar vertebrae in the neonate. The progressive increase in BMC and area from first to fourth lumbar vertebra is consistent with the anatomical changes of progressively larger size of lower lumbar vertebrae. However, the lack of progressive increase in areal BMD at each successive lumbar vertebra presumably reflects the biologic differences in the rate of change in area and $\mathrm{BMC}$, the basis for deriving the areal BMD. Our data therefore further supports the 
caution needed in the interpretation of DXA BMD in the growing skeleton. Whether there is a progressive change in true volumetric density at each lumbar vertebra is not known.

Ability to obtain DXA measurements for individual vertebra offers the opportunity to study selected changes under physiologic and pathologic conditions. However, DXA measurement of total bone mass of several vertebrae is probably more consistently reproducible than individual vertebra since bone mass of individual vertebra is significantly less than the total bone mass of several vertebrae, and any error in DXA measurement is likely to be proportionately greater if former is used as a basis for comparison.

There are limited reports on bone mineral status at lumbar vertebrae in healthy neonates particularly the preterm infants $(3,19,20)$. Different modes of data presentation based on first to fourth vertebrae as in this study and by others (20) or based on first to fifth lumbar vertebrae $(3,19)$ have been reported although the commercial software as used for this study allows only analyses of first to fourth lumbar vertebrae.

We conclude that DXA measurements of lumbar spine can be performed even in small preterm infants during newborn period. DXA LS measurements with its excellent precision in infants, low radiation exposure and rapid scan acquisition offer promise as a useful tool for widespread use in pediatrics. Our data may be used as a basis for further studies in physiologic and pathologic situations that may affect bone mineralization in infants.

\section{REFERENCES}

1. Koo WWK, Massom LR, Walters J 1995 Validation of accuracy and precision of dual energy X-ray absorptiometry for infants. J Bone Mineral Res 10:1111-1115

2. Picaud JC, Rigo J, Nyamugabo K, Milet J, Senterre J 1996 Evaluation of dual energy $\mathrm{X}$ ray absorptiometry for body composition assessment in piglets and term human neonates. Am J Clin Nutr 63:157-163

3. Braillon PM, Salle BL, Brunet J, Glorieux FH, Delmas PD, Meunier PJ 1992 Dual energy X-ray absorptiometry measurement of bone mineral content in newborns: Validation of the technique. Pediatr Res 32:77-80
4. Koo WWK, Walters J, Bush AJ, Chesney RW, Carlson SE 1996 Dual energy x-ray absorptiometry studies of bone mineral status in newborn infants. J Bone Mineral Res 11:997-1002

5. Koo WWK, Bush AJ, Walters J, Carlson SE 1998 Postnatal development of bone mineral status during infancy. J Am Coll Nutr 17:65-70

6. Brenner WE, Edelman DA, Hendricks CH 1976 A standard of fetal growth for the United States of America. Am J Obstet Gynecol 126:555-564

7. Ballard JL, Khoury JC, Wedig K, Wang L, Eilers-Walsman BL, Lipp R 1991 New Ballard score, expanded to include extremely premature infants. J Pediatr 119:417423

8. Bland JM, Altman DG 1986 Statistical methods for assessing agreement between two methods of clinical measurement. Lancet 1:307-310

9. Koo WWK, Walters J, Bush AJ 1995 Technical considerations of dual energy x-ray absorptiometry-based bone mineral measurements for pediatric studies. J Bone Mineral Res 10:1998-2004

10. Horwitz EM, Prockop DJ, Fitzpatrick LA, Koo WW, Gordon PL, Neel M, Sussman M, Orchard P, Marx JC, Pyeritz RE, Brenner MK 1999 Transplantability and therapeutic effects of bone marrow-derived mesenchymal cells in children with osteogenesis imperfecta. Nature Med 5:309-313

11. Koo WWK, Steichen JJ 1998 Osteopenia and Rickets of Prematurity. In Polin R, Fox W (eds), Fetal and Neonatal physiology, 2nd Ed. W.B. Saunders Company, Philadelphia, pp 2335-2349

12. Nelson DA, Koo WWK 1999 Interpretation of absorptiometric bone mass measurements in the growing skeleton: issues and limitations. Calcif Tissue Int 65:1-3

13. Prentice A, Parsons TJ, Cole TJ 1994 Uncritical use of bone mineral density in absorptiometry may lead to size-related artifacts in the identification of bone mineral determinants. Am J Clin Nutr 60:837-842

14. Trotter M, Hixon BB 1974 Sequential changes in weight, density, and percentage ash weight of human skeletons from an early fetal period through old age. Anat Rec 179:1-18

15. Namgung R, Tsang RC, Specker BL, Sierra RI, Ho ML 1994 Low bone mineral content and high serum osteocalcin and 1,25-dihydroxyvitamin D in summer- versus winter-born newborn infants: An early fetal effect? J Pediatr Gastroenterol Nutr 19:220-227

16. Gilsanz V, Roe TF, Mora S, Costin G, Goodman WG 1991 Changes in vertebral bone density in black girls and white girls during childhood and puberty. N Engl J Med 325:1597-1600

17. Ortiz O, Russell M, Daley TL, Baumgartner RN, Waki M, Lichtman S, Wang J, Pierson RN Jr, Heymsfield SB 1992 Differences in skeletal muscle and bone mineral mass between black and white females and their relevance to estimates of body composition. Am J Clin Nutr 55:8-13

18. Bonjour JP, Theintz G, Buchs B, Slosman D, Rizzoli R 1991 Critical years and stages of puberty for spinal and femoral bone mass accumulation during adolescence. J Clin Endocrinol Metab 73:555-563

19. Salle BL, Braillon P, Glorieux FH, Brunet J, Cavero E, Meunier PJ 1992 Lumbar bone mineral content measured by dual energy $\mathrm{X}$ ray absorptiometry in newborns and infants. Acta Pediatr 81:953-958

20. Tsukahara H, Sudo M, Umezaki M, Fujii Y, Kuriyama M, Yamamoto K, Ishii Y 1993 Measurement of lumbar spine bone mineral density in preterm infants by dual energy $\mathrm{X}$ ray absorptiometry. Biol Neonate 64:96-103 